\title{
To Study the utility of whats app application on smart phone in the department of neurosurgery
}

\begin{abstract}
Introduction: Before Whats App application on smart phone we used to communicate on phone and would discuss radiology on phone, there was no visual feedback and in some cases on call junior resident would miss some important radiological findings. This was a verbal communication and one to one communication. By our new system any case would be discussed among the team members and the chances of missing some radiological findings would be eliminated, patient management would be better and faster.
\end{abstract}

Objective: To determine the utility of smart phone and its Whats App application as a means of communication among the team members of Neurosurgery Department for better and quick patient management.

Materials and methods: From September 2012 till date, the authors used smart phones and its Whats App application as a means of communication among the team members for patient management and as an academic tool.

Results: From September 2012 there were 640 events related to patient care and management which were managed timely and efficiently by Whats App application. The opinion was sought from team members including the rotating residents regarding the utility of this application. Majority of them were satisfied with this method of the communication.

Conclusion: Smart phone and its Whats App application is an efficient means of communication for patient management, serves as a good academic tool, is very fast, easy and cheap.

Keywords: communication method; smart phone; whats app
Volume I Issue 2 - 2014

\author{
Anil Dhar, Rahul Gupta, Manish Vaish, Furqan \\ A Nizami, Manish Marda, Kapil Jain, Deepak \\ Varshney, Arvind Pal Singh \\ Anil Dhar, Rahul Gupta, Manish Vaish, Furqan A Nizami, Manish \\ Marda, Kapil Jain, Deepak Varshney, Arvind Pal Singh
}

Correspondence: Furqan A Nizami, Fortis Hospital, B-22 Sector 62 Noida, Gautam Budh Nagar, Uttar Pradesh, India, Tel +91-9797635348, Email drnizamifurqan@yahoo.co.in

Received: June II, 2014 | Published: June 20, 2014

\section{Introduction}

For communication between the members of any division in the surgical field, perhaps the most frequently used method is a verbal report via telephones. Telephone communication can be rapid but not objective and precise. To add more accurate information to the verbal report, clinical photographs taken by digital cameras were transmitted as downloadable files between computers having modem and telephone link as telemedicine has gained popularity in the late 1990's. ${ }^{1}$

The development of smart phones technology, with mobile high speed internet access and high resolution photographic capability has triggered a new era of real time communication in healthcare. With advances in internet access in late 2000s, images were transferred through internet lines by commonly used internet portals accessed by personal computers or by mobile phones. More recently photographs taken by smart phone were transferred to another smart phone. ${ }^{2,3}$

Whats App is an early stage technology startup founded to build a better short message service alternative. It is a proprietary cross platform instant messaging application for smart phones. Santa Clara, California based company Whats App Inc. that was founded in 2009 by Brian Acton \&Jan Koum ${ }^{4}$ developed it. In this study, we discussed our communication method reflecting the latest mobile environment using a smart phone and Whats App application. In addition, we analyzed the influence of this method on the time taken (Table 1) for managing any untoward incident happening in patient management.

Table I Time taken by second on call to react

\begin{tabular}{lll}
\hline Time Taken by Second on Call to Respond & Number of Episodes $(\mathbf{n}=\mathbf{6 4 0})$ & Percentage (\%) \\
\hline$<1$ min & 390 & 60.93 \\
I to $5 \mathrm{~min}$ & 200 & 31.25 \\
5 to $10 \mathrm{~min}$ & 38 & 5.93 \\
$>10 \mathrm{~min}$ & 12 & 1.87 \\
\hline
\end{tabular}

\section{Materials and methods}

This study was conducted in Neurosurgery Department, Fortis Hospital Noida and UP, India. The older methods of communication (verbal communication on phone) among the team members was replaced by latest Whats App application on smart phone Author started as a neurosurgeon group on Whats app. All team members

like senior consultants, consultant, senior residents, consultant anesthesiologist and junior residents (on rotation) were included in the group. All team members were well informed about the patients admitted at particular time, new admissions, patients planned for surgery, their pre operative plan, type of surgery. Pre operative and post operative images were uploaded and discussed among the 
team members, The junior resident uploaded the images and small history of the patient and the senior consultant on call wound respond immediately and would discuss the management of the patient with other team members, other members of the team would also give their opinion regarding the case. If in case the senior consultant on call would not reply he was contacted on the phone by on call junior resident or on call consultant and would discuss the management of the patient. In case of emergency admissions the senior consultant on call would decide about the patients who needed urgent surgical intervention, consultant anesthesiologist would also respond and advice about the investigations and any other consultations required before surgery. The junior resident on call would prepare the patient for surgery thus making it a faster method.

Intra operative short videos of surgeries which were of academic importance, Short video clips of the ambulation in post operative period, picture of condition of the operative site wound at the time of discharge was uploaded. Any untoward event was recorded and any academic activity planned was informed.., Patient's identity was not revealed at any stage. Patients were discussed according to the diagnosis, room number, whether operated or conservative, consultant in charge. After the morning rounds the new patients who were admitted, their imaging and management was discussed, any new information about already admitted patients was reviewed. All the members of the team would keep the notification on on Whats App, so that any information passed by any member would be received by all members of the team and the treatment would be initiated without delay, thus making it a real time communication method. Sometimes small video clips and audio was also attached word chat

All members downloaded Whats App from play store for android and app store for iOS. All team members had $3 \mathrm{G}$ internet services on their smart phone and all members were instructed to keep Whats App application on a notification on mode with a sound alert in order to respond immediately.

The chat conversations were photographed as screen shots and reviewed by author for study purposes and were analyzed on weekly basis. The on call junior resident would document the discussion and management on patient's file. At the end of the study the senior and junior residents were given questionnaire about the utility of the method of the communication.

\section{Discussion}

The time interval between patients admission and initiation of management is important in Neurosurgical patients and outcome also depends on how fast the treatment has been initiated (surgery or conservative) and also the time lag between any complication and initiation of any treatment is also important (Table 2). Our method of the communication has reduced this time interval. Before this system we used to communicate on the phone and would discuss radiology on phone and there was no visual feedback and in some cases on call junior resident would miss some important radiological findings. This was a verbal communication and one to one communication. By our new system any case would be discussed among the team members and the chances of missing some radiological findings would be eliminated, complication would be better, dealt with, wound care would also be better. Informed consent was taken from all patients for clicking photographs and sharing the information with the other members of the team. As only the team members were involved, so there were no adverse effects of the medico legal aspects of this tool.

Table 2 Time taken between detecting a problem and initiation of treatment

\begin{tabular}{lll}
\hline Time Taken Between Detecting A Problem and Initiation of Treatment & Number of Episodes $(\mathbf{n}=\mathbf{6 4 0})$ & Percentage (\%) \\
\hline$<5 \mathrm{~min}$ & 55 & 8.59 \\
5 to $10 \mathrm{~min}$ & 364 & 56.87 \\
10 to $15 \mathrm{~min}$ & 181 & 28.28 \\
$>15 \mathrm{~min}$ & 40 & 6.25
\end{tabular}

The disadvantages of this means of communication is that the chat system cannot replace verbal conversation in case of critical cases and discussion is based on single image and multiple images need to be transferred in order to get a clear picture of the patient. The short coming of this tool is that all members have to remain logged on to internet round the clock. , that conversation cannot form the part of medical record, as it cannot be printed directly and while discussing multiple patients there might be some confusion about which patient is being discussed unless there are enough identifying features of the patient.

We reviewed the literature to find if this new method has been used before in any neurosurgical field, but there was no reported evidence. In Neurosurgery literature image transfer through mobile phone has been used and found effective. ${ }^{5}$

Innovation similar to this modality discussed in this report have been used earlier in interpreting radiological images in the emergency room, which used images displayed on a picture achieving and communication system monitor captured by a camera and transferred to remote physicians through wireless higher bandwidth network. ${ }^{6}$
Since in India there is scarcity of Neurosurgeon, so one Neurosurgeon has to take care of multiple hospitals, so keeping this in mind it becomes easier for a Neurosurgeon to manage the patients in different hospitals at a particular point of time.

In addition this method has made life easier for both on call resident and on call consultant, the consultants need not be physically present and as such they can utilize this time for other patient care activities and academic activities. Majority of senior and junior resident were convinced that this system was effective teaching method in evaluation of patients and making available photographs and videos for presentation. Majority of the residents were happy and satisfied with this method of the communication and they would recommend this method to be used in other hospitals also.

This method can be used for seeking advice from the experts in a particular sub specialty who are living at a distance from the hospital without physically being present. This would help in better managing the patients and a better outcome. In future this can be used for management of trauma to start pre hospital management which can in turn lead to saving lives. 
The limitation of this study was that there was no control sample to compare speed of initiation of patient management in pre Whats App era, if there was one, we could see statistically difference between the two methods.

\section{Conclusion}

For quick communication among staff members, we used smart phone and What App chat application for patient care and academic support (Table 3). We found out that it was very effective and satisfying to all team members. Moreover, any notification of patient's events was quick and start of management was faster with this method. We recommend that this method should be used in other specialties for patient care and academic purposes. However one thing has to be kept in mind those messages of any sort cannot replace a personal conversation in case of critical issue.

Table 3 Feedback of doctors regarding utility of this mode of communication $(n=50)$

\begin{tabular}{llll}
\hline Topic for Feedback & Yes & No & No Comment \\
\hline Excellent mode of communication & 45 & 4 & I \\
Will recommend this method of communication to be used in other departments & 40 & 8 & 2 \\
Cost effective & 42 & 7 & I \\
Faster method & 41 & 8 & I \\
Better for patient care and management & 46 & 3 & I \\
Reliable method & - & - & - \\
More knowledge about all the patients admitted at a particular time & 41 & 8 & I \\
Has eased presentations in grand rounds & 38 & 9 & 3 \\
Good academic tool & 37 & 10 & 3 \\
Extra burden as Doctor is occupied even in off hours & 5 & 43 & 2 \\
Best support when one or more Neurosurgeon is on leave & 45 & 4 & I \\
\hline
\end{tabular}

\section{Acknowledgments}

None.

\section{Conflicts of interest}

The authors declare there are no conflicts of interest related to the article.

\section{References}

1. Baldwin AJ, Langton SG. Postoperative monitoring of flaps by digital camera and Internet link. Br J Oral Maxillo fac Surg. 2001;39(2):120121.

2. Varkey P, Tan NC, Girotto R, et al. A picture speaks a thousand words: The use of digital photography and the Internet as a cost-effective tool in monitoring free flaps. Ann Plast Surg. 2008;60(1):45-48.
3. Engel H, Huang JJ, Tsao CK, et al. Remote real-time monitoring of free flaps via Smartphone photography and $3 \mathrm{G}$ wireless Internet: a prospective study evidencing diagnostic accuracy. Microsurgery. 2011;31(8):589-595.

4. http://en.wikipedia.org/wiki/Whats_App

5. Yamada M, Watarai $\mathrm{H}$, Andou T, et al. Emergency image transfer system through a mobile telephone in Japan: technical note. Neurosurgery. 2003;52(4):986-990.

6. Kim DK, Yoo SK, Kim SH. Instant wireless transmission of radiological images using a personal digital assistant phone for emergency teleconsultation. $J$ Telemed Telecare. 2005;11(Suppl 2):S58-S61. 